\title{
Paper
}

\section{Stabilization Effect of Self-Assembly Dendrimer Doped Cholesteric Liquid Crystal on Helical Structure}

\author{
Takumi Shiomi $^{\dagger}$, Yosei Shibata ${ }^{\dagger}$, Takahiro Ishinabe ${ }^{\dagger}$ (member) and Hideo Fujikake ${ }^{\dagger}$ (fellow) \\ Abstract In this paper, we report the resettable functionality of helix pitch length in the mixture composed \\ of cholesteric liquid crystal and self-assembly dendrimer with lysine groups. Cholesteric-phase liquid crystal was \\ obtained by doping a tiny amount of dendrimer less than $0.01 \mathrm{wt} \%$ concentration. The mixture showed not only \\ thermal stability of helix pitch length but also resettability of that by temperature control process based on sol- \\ gel transition characteristics in the dendrimer with lysine group. We discuss the temperature effect of the \\ proposed cholesteric liquid crystal on such unique functionality.
}

Keywords: cholesteric liquid crystal, self-assembly dendrimer, thermal stabilization, helix pitch length, molecular doping.

\section{Introduction}

Cholesteric-phase liquid crystal (CLC) is composed of the nematic-phase liquid crystalline material and chiral dopant and has molecular alignment having helical configuration. When this helical configuration shows one-dimensional periodic structure between two substrates, CLC reflects the circularly-polarized light of a specific wavelength corresponding to helix pitch length; called Bragg's reflection ${ }^{1)}$. The wavelength of reflected light can be any controlled by helix pitch length. This feature leads to the development of many optoelectronic applications so far, such as wavelengthtunable lasers ${ }^{2}$, optical recording materials ${ }^{3)}$, optical shutters ${ }^{4)}$, and full-colored electronic paper ${ }^{5)}$. Although CLC materials have attractive characteristics, there is a drawback of instability of helical structure to environmental temperature changes in case of weak helical twisting power (HTP). The HTP value of chiral dopant decreases with an increase in temperature. For this reason, improvement of thermal stabilization in CLC is necessary for every application.

To overcome this issue, stabilized helical structure of CLC using photo-polymerized monomer as the additive has been studied in many cases ${ }^{6-10)}$ (see Fig.1(a)). Of these kinds of literature, $\mathrm{R}$. Guo et al. reported the stabilization principle of CLC using polymers as

Received April 1, 2021; Revised May 31, 2021; Accepted June 25, 2021

$\dagger$ Department of Electronics, Graduate School of Engineering, Tohoku

University

(Sendai, Japan)

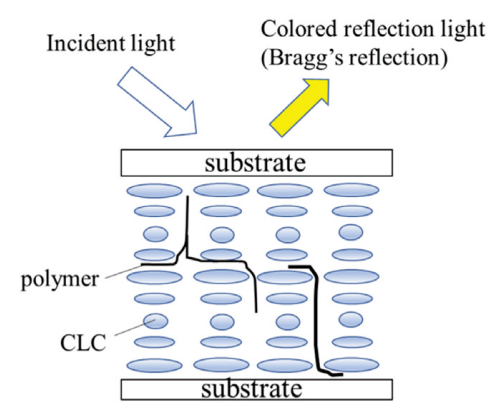

(a)

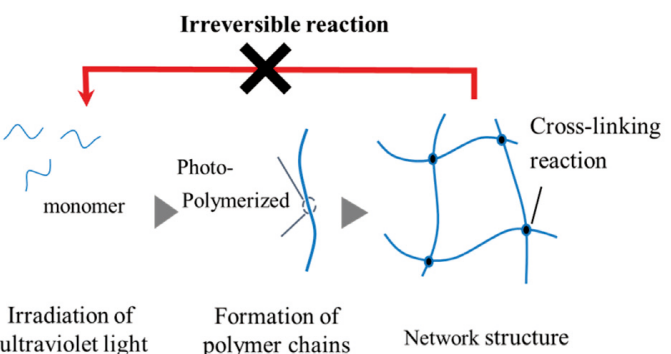

(b)

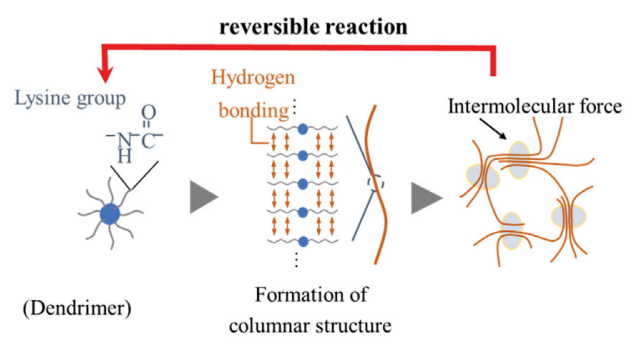

(c)

Fig.1 (a) Schematic illustration of polymer stabilized cholesteric liquid crystal (b) photo-polymerization reaction of monomer (c) aggregation of self-assembly dendrimers. 
follows ${ }^{10)}$. First, the formation of polymer network structure was occurred by irradiation of ultraviolet light to the mixture of CLC and monomer. The polymer network partly formed in the void among helical structures and behaves as the base. The molecular alignment of the entire CLC is stabilized by elastic force among liquid crystal molecules (see Fig.1 (b)). Besides, the bandwidth of reflection spectra can be tuned by temperature control at ultraviolet light irradiation due to the temperature dependence of HTP value on chiral dopants ${ }^{10)}$. There is a possibility that the unique functionality in a mixture of CLC and other materials opens the door for the novel device application.

In this paper, we proposed the mixture of CLC and self-assembly dendrimer with lysine groups for exploration of the unique functionality in CLC. Previous reports clarified that the dendrimer molecules in nematic-phase LC enable the formation of nano-sized fibril and self-aggregated fibrils (network structure) ${ }^{11}$, 12). Besides, we clarified that this dendrimer has an anchoring effect on liquid crystalline molecules ${ }^{13}$. Interestingly, the dendrimer enables the reconstruction of fibril or network structure by temperature control ${ }^{14)}$. This reconstruction characteristic is based on reversible hydrogen bonding among self-assembly lysine groups ${ }^{11}$, 12), as shown in Fig.1 (c). The difference from conventional polymer stabilization is aggregation with reversible reaction. As the advantage of these characteristics, stabilization and reset functionalities of helix pitch length are expected.

Here, we report on the temperature control effect of the mixture of CLC and self-assembly dendrimer on helix pitch length.

\section{Proposed Method for CLC Stabilization}

The operation principle of the proposed method using self-assembly dendrimers with lysine groups (POSS-Lys) is as follows. According to the literature 11-14), this dendrimer can be formed a nano-sized aggregation structure in nematic-phase liquid crystals. The concentration of POSS-Lys over $0.5 \mathrm{wt} \%$ induces randomly-aligned LC and light scattering state ${ }^{13)}$. This fact means the presence of molecular interaction between POSS-Lys and LC. The concentration of doping level will be desirable for stabilization of helix structure in CLC, less than $0.5 \mathrm{wt} \%$ at least. The optimal concentration of POSS-Lys was discussed in section 4.1. The CLC alignment stabilizes by dispersed POSS-Lys aggregates.

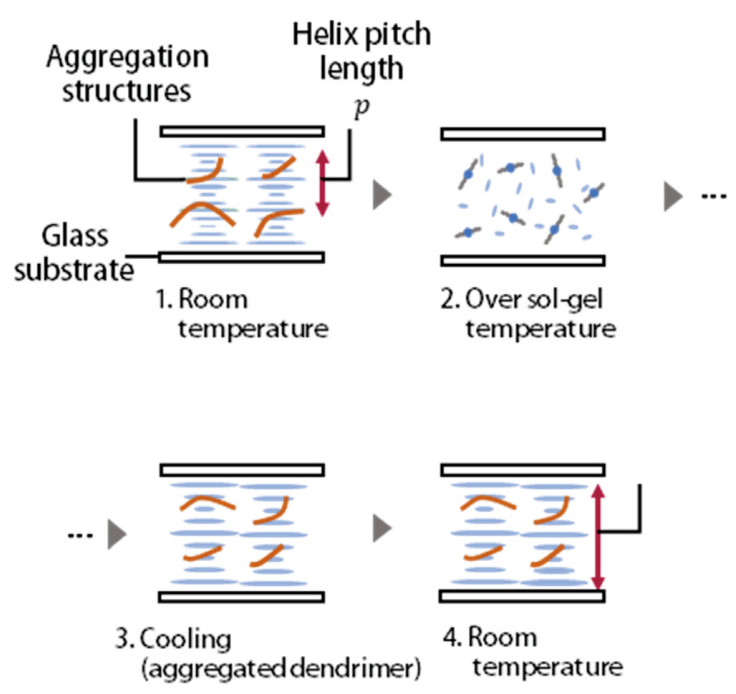

Fig.2 Schematic illustration of proposed control method of helix pitch length using self- alignment of that liquid crystals assembled dendrimer.

Furthermore, this dendrimer can be dispersed on the individual molecular level by thermal treatment over sol-gel transition temperature which is reported as about $120{ }^{\circ} \mathrm{C}$. The cooling process after heating over solgel transition induces the reconstruction of the aggregated POSS-Lys molecules because aggregation among POSS-Lys is the reversible reaction as mentioned above. The resettable functionality of the helix structure will be useful for unintentional temperature and external mechanical stress to CLC devices. The mentioned above principle summarized Fig. 2. In this paper, we investigated the stability and resettability of the helix structure.

\section{Experimental}

\subsection{Materials}

In this study, we used E-7 (purchased from LCC Corp.) as a nematic-phase liquid crystal with cyano groups. The chiral dopant as the additive for CLC formation was DDS-1015L (purchased from LCC corp.). The amount of chiral dopant was uniformly set to $6 \mathrm{wt} \%$ regardless of the concentration of POSS-Lys (synthesized from Nard Institute Ltd.). In the preparation of the wedge-type cell, we used AL1254 (purchased from JSR corp.) as the alignment film, and rubbing treatment was performed on the anti-parallel. The prepared liquid crystals were injected into the wedge-type cell and observed under Crossed-Nicols polarizing microscope.

\subsection{Estimation of Helix Pitch Length}

To determine the helix pitch length of fabricated CLC, we used a wedge-type cell as a reference of the literature ${ }^{15}$. 


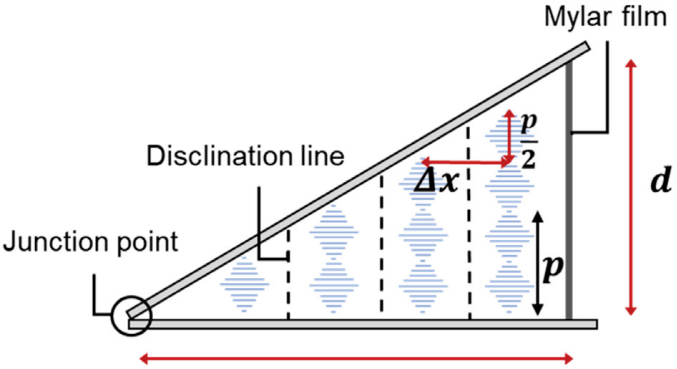

$\boldsymbol{L}$

Fig.3 Schematic diagram of the wedge-type cell structure.

Fig. 3 shows the schematic of the wedge-type cell. A film spacer with a thickness of several tens' micrometers was used as a spacer on only one side of the glass substrate. In such configuration, the upper glass substrate is tilted and the thickness of the liquid crystal layer is distributed. When the CLC is injected into the cell, disclination lines appear where the helix angle changes by $180^{\circ}$. The ratio of the distance $\Delta x$ between the disclination lines and the half-pitch of the CLC is equal to the ratio of the distance $L$ from the junction point of the glass substrate to the spacer and the thickness $d$ of the spacer. Here, we defined helix pitch length as $\boldsymbol{p}$. The $\boldsymbol{p}$ value can be evaluated by Equation (1).

$$
p=2 \frac{d \Delta x}{L}
$$

Since $d$ and $L$ values are known from the fabrication condition of the wedge-type cell, it is possible to evaluate the helix pitch length $\boldsymbol{p}$ by measuring the distance $\Delta \boldsymbol{x}$ between the disclination lines.

\section{Results}

\subsection{Suitable concentration of dendrimer}

In this study, POSS-Lys (synthesized from Nard institute ltd.) was used as a self-assembly dendrimer material. According to the related literature, this material has an interesting ability: nano-sized fibril structure was formed and enables reconstruction of its structure even if such structure was destroyed. ${ }^{16)}$. The molecular structure of POSS-Lys is shown in Fig. 4. We have reported that when $0.50 \mathrm{wt} \%$ of POSS-Lys is added to a nematic-phase liquid crystal having a cyanide group, the liquid crystal is randomly aligned due to gelation ${ }^{13)}$. However, maintaining the molecular alignment of the liquid crystal is required to form the helix structure. Therefore, we investigated the appropriate amount of dendrimer to be added to control the alignment of CLC. We used E-7 (purchased from

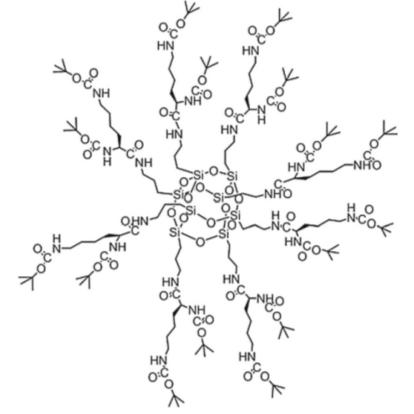

Fig.4 The molecular structure of POSS-Lys.

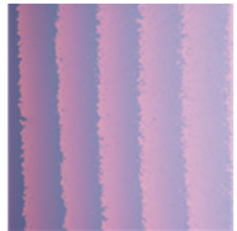

(a)

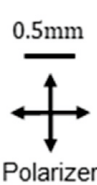

Polarize

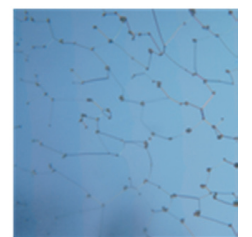

(b)
Fig.5 Photograph of a wedge-type cell with CLC in CrossedNicols. (a) The concentration of POSS-Lys: $0.01 \mathrm{wt} \%$ (b) The concentration of POSS-Lys: $0.50 \mathrm{wt} \%$.

LCC) terminally modified with cyano groups as the nematic liquid crystal and DDS-1015L (purchased from LCC) as the chiral dopant. We injected the prepared liquid crystals into a wedge cell. Then the disclination lines were observed using a polarizing microscope in a Crossed-Nicols environment. The results are shown in Fig. 5. When $0.50 \mathrm{wt} \%$ of POSS-Lys was doped into the CLC, no disclination lines were observed and the alignment of the liquid crystal was not confirmed. When the number of POSS-Lys was gradually reduced to 0.01 wt $\%$, the disclination lines were observed, indicating that the alignment of the liquid crystal was maintained. The concentration of $0.01 \mathrm{wt} \%$ is extremely low. Therefore, the network structure might not be built up enough to prevent the alignment of the liquid crystal, and the aggregation has progressed only to the level of short fiber fragments.

\subsection{Stabilization Effect of Helix Pitch}

The addition of dendrimers to CLC may suppress the change in helix pitch length with temperature change. Here, we investigated the stabilizing effect of doping 0.01 wt\% concentration of POSS-Lys on the helix pitch length in CLC. The evaluation process is shown in Fig. 6. CLC containing $0.01 \mathrm{wt} \%$ POSS-Lys was injected into the wedge-type cell. The individual POSS-Lys molecules are then dispersed by heating to $150{ }^{\circ} \mathrm{C}$, over the sol-gel transition temperature. To promote the aggregation of POSS-Lys, the cell was slowly cooled to room 


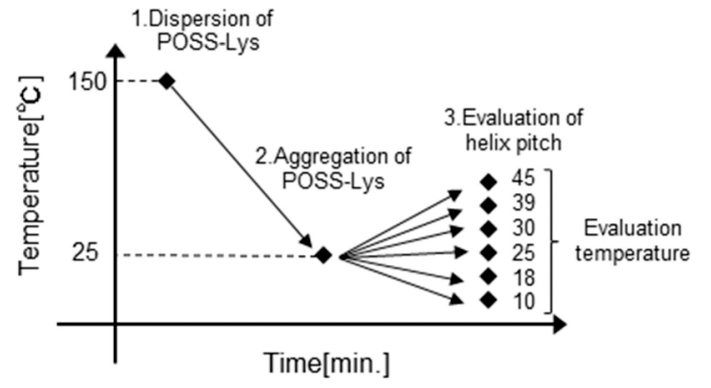

Fig.6 Schematic diagram of the temperature changing process of evaluation of the helix pitch stabilization.

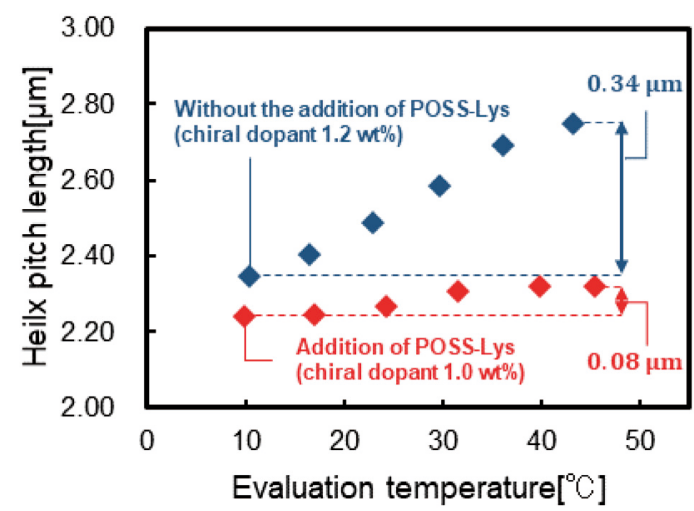

Fig.7 Dependence of helix pitch length on evaluation temperature changes under different concentration of chiral dopant. (concentration of dendrimer: $0.01 \mathrm{wt} \%$ ).

temperature over 10 minutes and left to stand for 15 minutes. The temperature was then changed over 10 minutes to the evaluation temperature, and the helix pitch length was evaluated at each temperature. CLC is known that the helix pitch length changes when other impurities are doped. The amount of chiral dopant doped into the CLC was adjusted to compare the change in the helix pitch length. Here, the amount of chiral dopant was determined so that the helix pitch length at $10{ }^{\circ} \mathrm{C}$ would be equal. This decision was made to standardize the starting time of the change in helix pitch length.

Fig. 7 shows the results of the temperature dependence of the helix pitch length. In the case of data with POSS-Lys, the variation of helix pitch length from $10{ }^{\circ} \mathrm{C}$ to $45{ }^{\circ} \mathrm{C}$ was $0.08 \mu \mathrm{m}$. In the case of without POSS-Lys, the variation of helix pitch length from $10{ }^{\circ} \mathrm{C}$ and $45{ }^{\circ} \mathrm{C}$ was $0.34 \mu \mathrm{m}$. Compared to the case without POSS-Lys, the change in helix pitch length was significantly suppressed. This result indicates that the dendrimer has the effect of stabilizing the helix pitch length because the amount of pitch change differs depending on the presence or absence of the dendrimer.

\subsection{Resettable Functionality of Helix Pitch}

POSS-Lys undergoes a bond between molecules on

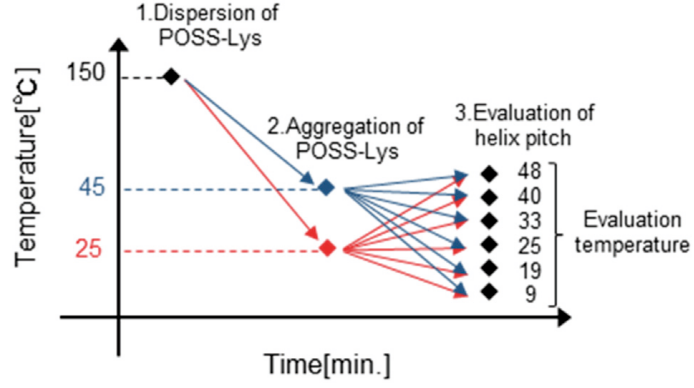

Fig.8 Schematic diagram of the temperature changing process of evaluation of the helix pitch resettability.

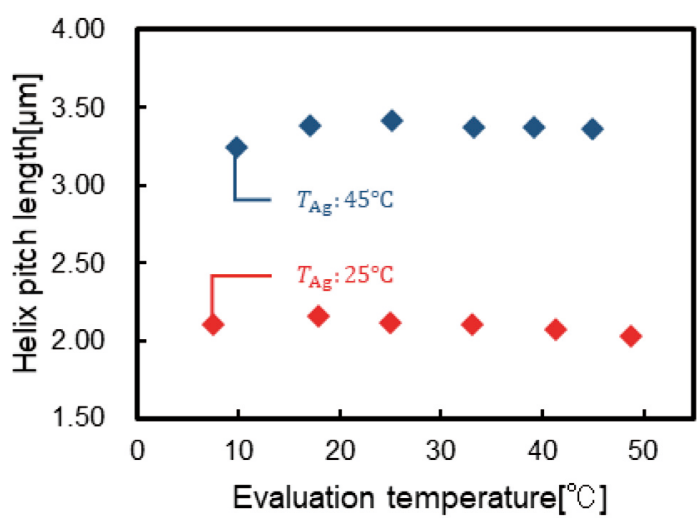

Fig.9 Comparison of fixed helix pitch length with changes in aggregation temperature.

putting quietly, it can be promoted aggregation. This bond originates from the hydrogen-bonding functional group on the side chain, which can be dissolved by applying high heat. This property has been reported to enable the construction and reconstruction of aggregation structures. We expect to be able to fix the helix pitch length to an arbitrary length by determining the temperature of the static setting (defined as the aggregation temperature $T_{\mathrm{Ag}}$ ) that promotes the aggregation of POSS-Lys.

Therefore, we evaluated the fixed helix pitch length by changing $T_{\mathrm{Ag}}$. The cell was heated up to $150{ }^{\circ} \mathrm{C}$ to separate the dendrimer molecules, then slowly cooled to $T_{\text {Ag }}\left(25{ }^{\circ} \mathrm{C}, 45^{\circ} \mathrm{C}\right)$ and allowed to stand. The helix pitch length was then evaluated at each evaluation temperature (see Fig. 8).

The results are shown in Fig. 9. As shown in Fig. 9, the fixed helix pitch length can be successfully changed by changing $T_{\mathrm{Ag}}$. This result indicates that the helix pitch can be re-set by controlling the temperature, and the reflective band can be switched intentionally. 


\section{Discussion}

\subsection{Stabilization Mechanism of Helix Pitch Length}

When the amount of dendrimer added is $0.01 \mathrm{wt} \%$, the network structure is considered not to have been established because the alignment of the liquid crystal can be maintained. In this case, the dendrimers are expected to be either dispersed in the liquid crystal or aggregated to the level of short fiber fragments. As shown in Fig. 9, the addition of dendrimers enabled the resetting of the helix pitch length. In this process, it needs to be heated up to the sol-gel transition temperature. If the dendrimers do not aggregate and are dispersed in the liquid crystal, heating to this temperature would not be necessary, so we guess that the aggregation of the dendrimers has progressed to the level of short fibers.

Based on the fact that the aggregation structure of the short fiber fragments is constructed, the stabilization mechanism of the helix pitch length will be discussed. As shown in Fig. 7, the helix pitch length was stabilized by the addition of POSS-Lys. A change in the helix pitch length means a change in the degree of twist of the liquid crystal molecules. Therefore, the alignment of the liquid crystal molecules is significantly involved in the stabilization of the helix pitch length. The existence of intermolecular interactions between POSS-Lys and liquid crystal molecules that affect the alignment has already been reported ${ }^{13)}$. Liquid crystal molecules induced a helical structure by chiral dopant is distributed around the aggregation structure of the POSS-Lys, interaction occurs between the molecules. This intermolecular interaction is presumed to stabilize the alignment of the liquid crystal molecules and suppress the variation in the degree of twist. In the future, we plan to conduct experiments to prove this assumption.

\subsection{Mechanism of re-setting the Helix Pitch Length}

The helix pitch length of CLC is known to vary with temperature. The helix pitch length is fixed at the point when the aggregation of POSS-Lys is completed. By selecting the $T_{\mathrm{Ag}}$, it can be changed to an arbitrary helix pitch length. By leaving it at that temperature, the helix pitch length is stabilized by the aggregation structure of POSS-Lys. Therefore, changing the $T_{\mathrm{Ag}}$ is expected to change the fixed helix pitch length. As shown in Fig. 7, the helix pitch length of CLC without POSS-Lys increased with increasing evaluation temperature. Since the helix pitch length is fixed by $T_{\mathrm{Ag}}$, the higher the $T_{\mathrm{Ag}}$, the longer the helix pitch is fixed. These results indicate that the fixed helix pitch length changes when the aggregation temperature of POSS-Lys is changed. We believe that this research has the potential to expand the application possibilities of CLC and to be involved in the development of new devices.

\section{Conclusion}

In this paper, we proposed a method to control the helix pitch length of CLC doped with dendrimers. We showed that the helix pitch length can be fixed by doping 0.01 wt\% concentration of self-assembly dendrimers into the CLC. We have also shown that the helix pitch length can be freely selected by changing the aggregation temperature of the dendrimer.

\section{References}

1) R. Dreher and G. Meier: "Optical properties of cholesteric liquid crystals", Phys. Rev. A, 8, 3, pp.1616-1623 (1973)

2) J. Schmidtke, W. Stille, H. Finkelmann and S.T. Kim: "Laser emission in a dye doped cholesteric polymer network" , Adv. Mater., 14, 10, pp.746-749 (2002)

3) N. Tamaki: "Cholesteric Liquid Crystal Recording by Thermochromism and Photochromism," 45, 10, pp.533-538 (2007)

4) D.K. Yang, L.C. Chien and J.W. Doane: "Cholesteric liquid crystal/polymer dispersion for haze-free light shutters," Appl. Phys. Lett., 60, 25, pp.3102-3104 (1992)

5) Y. Kageyama: "Now and Future of Color Electric Paper Introduction to Cholesteric Liquid Crystal e-paper," The Japanese Society of Printing Science and Technology, 44, 5, pp.275-278 (2007)

6) S. Relaix, C. Bourgerette and M. Mitov: "Broadband reflective liquid crystalline gels due to the ultraviolet light screening made by the liquid crystal," Appl. Phys. Lett.,89, 25 (2006)

7) R.A. M. Hikmet and R. Polesso: "Patterned multicolor switchable cholesteric liquid crystal gels," Adv. Mater., 14, 7, pp.502-504 (2002)

8) Z. Bian, K. Li, W. Huang, H. Cao, H. Yang and H. Zhang: "Characteristics of selective reflection of chiral nematic liquid crystalline gels with a nonuniform pitch distribution," Appl. Phys. Lett., 91, 20 (2007)

9) O.G. Mamack. Marc, Coombs. Neil: "Polymer Network - Stabilized Liquid Crystals," Adv. Mater., 12, p. 167 (2000)

10) R. Guo, K. Li, H. Cao, X. Wu, G. Wang, Z. Cheng, F. Wang, H. Zhang and H. Yang.: "Chiral polymer networks with a broad reflection band achieved with varying temperature," Polymer (Guildf)., 51, 25, pp.5990-5996 (2010)

11) S. Chen, L. Zhou, Z. An, H. He, M. Ma, Y. Shi and X. Wang.: "Driving force balance - The 'identity card' of supramolecules in a self-sorting multicomponent assembly system," Soft Matter, 17, 1, pp.153-159 (2021)

12) G. Tang, S. Chen, F. Ye, X. Xu, J. Fang and X. Wang: "Loofah-like gel network formed by the self-assembly of a $3 \mathrm{D}$ radially symmetrical organic-inorganic hybrid gelator," Chem. Commun., 50, 54, pp.7180-7183 (2014)

13) R. Saito, Y. Shibata, T. Ishinabe and H. Fujikake: "Electro-optical characteristics and curvature resistance of dye-doped liquid crystal gel films for stretchable displays," IEICE Trans. Electron., E101C, pp.901-905 (2018) 
14) Y. Shibata, R. Saito, T. Ishinabe and H. Fujikake: "Mechanical stability and self-recovery property of liquid crystal gel films with hydrogen-bonding interaction," IEICE Trans. Electron., E102C, 11, pp.813-817 (2019)

15) K. Matsumura and S. Iwayanagi: "Determination of Helical mea and Optical Rotatory Power of a Mixed Cholesteric Liquid Crystal by Cano Wedge Arrangement," Appl. Phys, 43, 2, pp.126-131 (1974)

16) H. He, S. Chen, X. Tong, Z. An, M. Ma, X. Wang and X. Wang.: "Self-Assembly of a Strong Polyhedral Oligomeric Silsesquioxane Core-Based Aspartate Derivative Dendrimer Supramolecular Gelator in Different Polarity Solvents," Langmuir, 33, 46, pp.13332-13342 (2017)

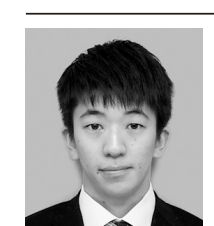

Takumi Shiomi received a B.E. degree from Tohoku University, Sendai, Japan, in 2020. He is a Master's student in the Department of Electronic Engineering at the Graduate School of Engineering, Tohoku University. His research interest is the exploration of the novel application using structurallystabilized cholesteric liquid crystal.

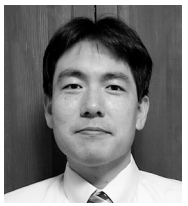

Yosei Shibata received M. E. degree from Yamagata University, Yamagata, Japan, in 2010, and the $\mathrm{Ph}$. D. degree in engineering from Tokyo Institute of Technology, Japan, in March, 2013. Then he joined the National Institute of Advanced Industrial Science and Technologies (AIST, Japan) as a postdoctoral position in Research Center for Photovoltaic Technologies and Flexible Electronics Research Center. In October 2015, he joined the Department of Electronics of Tohoku University as an Assistant Professor. His research interests on molecular-ordering technique and organic crystalline devices such as organic transistors and photovoltaics. He received the Young Scientist Paper Award from Japan Society of Applied Pysics (JSAP) in 2015 and Japan Liquid Crystal Society (JLCS) in 2020.

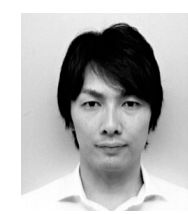

Takahiro Ishinabe received his B.S., M.S., and Ph. D. degrees in Electronic Engineering from Tohoku University, Sendai, Japan, in 1995, 1997 and 2000 , respectively. From 2000 to 2002 , he was a Research Fellow of the Japan Society for the Promotion of Science and from 2003 to 2012, he was an Assistant Professor, and since 2013, he has been an Associate Professor in the Department of Electronics, Graduate school of Engineering, Tohoku University. He has also been a Visiting Professor in the CREOL, The College of Optics and Photonics, University of Central Florida from 2010 to 2011 . He has been performing a research on advanced liquid crystal displays such as wide viewing angle LCD, reflective full-color LCD, field sequentialcolor LCD and flexible LCD.

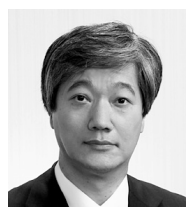

Hideo Fujikake received M.E and Ph.D. degrees from Tohoku University, Japan, in 1985 and 2003 , respectively. In 1985 , he joined the Japan Broadcasting Corporation (NHK). In 1988-2012, he worked for NHK Science and Technology Research Laboratories. From 2006, he was a visiting professor at Department of Physics, Graduate School of Science, Tokyo University of Science. Since 2012, he has been a professor at the Department of Electronic Engineering, Tohoku University. He received the Best Paper Award from the Institute of Electronics, Information and Communication Engineers (IEICE) in 2001 and 2017, the Best Paper Award from the Japanese Liquid Crystal Society (JLCS) in 2001, 2015 and 2020, the Niwa-Takayanagi Best Paper Award from the Institute of Image Information and Television Engineers of Japan (ITE) in 2003 and 2009, and the Electronics Society Award from IEICE in 2013. His current interests are concerned with flexible liquid crystal displays and functional optical devices including holography. He also served as a Japan Chapter Chair in IEEE Consumer Electronics Society in 2012-2014, a General Vice Chair of International Display Workshops from 2015-2016, and a Vice President of the Japanese Liquid Crystal Society from 2015-2016. He has been ITE, IEICE and JSAP (the Japan Society of Applied Physics) fellows. 